\title{
Correction: A qualitative transcriptional signature for determining the grade of colorectal adenocarcinoma
}

Hailong Zheng · Kai Song · Yelin Fu • Tianyi You • Jing Yang • Wenbing Guo · Kai Wang • Liangliang Jin • Yunyan Gu • Lishuang Qi · Wenyuan Zhao · Zheng Guo

Published online: 5 December 2019

(C) The Author(s), under exclusive licence to Springer Nature America, Inc. 2019

Correction to: Cancer Gene Therapy https://doi.org/10.1038/ s41417-019-0139-1, published online 09 October 2019

In the original version of this Article, the affiliation details for Department of Systems Biology, College of Bioinformatics
Science and Technology, Harbin Medical University, Harbin 150086, China, were not assigned to all of the authors. This has now been corrected in both the PDF and HTML versions of the Article. 